Comment. Math. Helv. 72 (1997) 426-433

(C) 1997 Birkhäuser Verlag, Basel

0010-2571/97/030426-08 \$1.50+0.20/0

Commentarii Mathematici Helvetici

\title{
Differential operators commuting with invariant functions
}

\author{
T. Levasseur and J. T. Stafford*
}

\begin{abstract}
Let $\mathfrak{g}$ be a reductive, complex Lie algebra, with adjoint group $G$, let $G$ act on the ring of differential operators $\mathcal{D}(\mathfrak{g})$ via the adjoint action and write $\tau: \mathfrak{g} \rightarrow \mathcal{D}(\mathfrak{g})$ for the differential of this action. We prove that the commutant, in $\mathcal{D}(\mathfrak{g})$, of $\mathcal{O}(\mathfrak{g})^{G}$ is the algebra generated by $\mathcal{O}(\mathfrak{g})$ and $\tau(\mathfrak{g})$, thereby answering a question of Barlet.
\end{abstract}

Mathematics Subject Classification (1991). 22E46, 14L30, 16S32, 17B20.

Keywords. Invariant differential operators, commuting differential operators, semi-simple Lie algebras, symmetric algebras.

\section{Introduction}

Fix a reductive, complex Lie algebra $\mathfrak{g}$, with adjoint group $G$, let $G$ act on the ring of differential operators $\mathcal{D}(\mathfrak{g})$ via the adjoint action and write $\tau: \mathfrak{g} \rightarrow \mathcal{D}(\mathfrak{g})$ for the differential of this action. We identify $\mathcal{O}(\mathfrak{g})$, the ring of regular functions on $\mathfrak{g}$, with $S\left(\mathfrak{g}^{*}\right)$ and let $\mathcal{O}(\mathfrak{g})^{G}$ denote the subalgebra of $G$-invariant functions. The aim of this note is to prove:

Theorem 1.1. The commutant $\mathcal{C}=\mathcal{C}_{\mathcal{D}(\mathfrak{g})}\left(\mathcal{O}(\mathfrak{g})^{G}\right)$, in $\mathcal{D}(\mathfrak{g})$, of $\mathcal{O}(\mathfrak{g})^{G}$ is the algebra generated by $\mathcal{O}(\mathfrak{g})$ and $\tau(\mathfrak{g})$.

At the level of vector fields, this result follows from [5, Theorem 2.1], in the sense that Dixmier's result implies that $\mathcal{C} \cap \operatorname{Der} \mathcal{O}(\mathfrak{g})=\mathcal{O}(\mathfrak{g}) \tau(\mathfrak{g})$. In [2], D. Barlet raised the question of whether Theorem 1.1 is true, since this would form a natural generalization of Dixmier's result. In the same paper, Barlet was able to prove the theorem in the case when $\mathfrak{g}=\mathfrak{g l}(n, \mathbb{C})$. We would like to thank M. Raïs for bringing Barlet's question to our attention.

In the process of proving Theorem 1.1, we obtain a considerable amount of information about the structure of $\mathcal{C}$. Some particular properties are given in the next result. The unexplained definitions can be found in Section 3.

*The research of the second author was supported in part by an NSF grant 
Proposition 1.2. $\mathcal{C}$ is an Auslander-Gorenstein, CM domain and a maximal order in its quotient division ring.

In fact, the main theorem of this paper is a result about commutative rings. To state this, let $A$ denote the subalgebra of $\mathcal{D}(\mathfrak{g})$ generated by $\mathcal{O}(\mathfrak{g})$ and $\tau(\mathfrak{g})$ and set $E=\mathcal{O}(\mathfrak{g}) \tau(\mathfrak{g}) \subset \operatorname{Der} \mathcal{O}(\mathfrak{g})$. If one filters $\mathcal{D}(\mathfrak{g})$ and its subalgebras by degree of differential operators, then it is easy to see that the associated graded rings gr $A$ and gr $\mathcal{C}$ are domains with the same quotient field as the symmetric algebra $\operatorname{Sym}_{\mathcal{O}(\mathfrak{g})}(E)$. Then, Theorem 1.1 and Proposition 1.2 follow easily from the following result.

Theorem 1.3. (i) Let $E=\mathcal{O}(\mathfrak{g}) \tau(\mathfrak{g}) \subset \operatorname{Der} \mathcal{O}(\mathfrak{g})$. Then $\operatorname{Sym}_{\mathcal{O}(\mathfrak{g})}(E)$ is a factorial, complete intersection of Krull dimension $2 \operatorname{dim} \mathfrak{g}-\mathrm{rk} \mathfrak{g}$.

(ii) $\operatorname{gr} A=\operatorname{gr} \mathcal{C}=\operatorname{Sym}_{\mathcal{O}(\mathfrak{g})}(E)$.

This research was conducted while the second author was visiting and partially supported by the University of Poitiers. He would like to thank that institution for its hospitality and financial support.

\section{The symmetric algebra of the module generated by $\tau(\mathfrak{g})$}

In this section we prove Theorem 1.3 from the introduction. We begin with some preliminary notation and results.

As before, we fix a complex, reductive Lie algebra $\mathfrak{g}$ of dimension $n$ and rank $\ell$. Write $G$ for the adjoint group of $\mathfrak{g}$. Define the categorical quotient of $\mathfrak{g}$ by $\mathcal{O}(\mathfrak{g} / / G)=\operatorname{Spec} \mathcal{O}(\mathfrak{g})^{G}$ and let $u: \mathfrak{g} \rightarrow \mathfrak{g} / / G$ denote the quotient morphism. We will write $\mathcal{O}$ for $\mathcal{O}(\mathfrak{g})$. Define

$$
X_{i}=\left\{y \in \mathfrak{g}: \operatorname{rk} d_{y} u \leq i\right\},
$$

where $d_{y} u: T_{y} \mathfrak{g} \rightarrow T_{u(y)} \mathfrak{g} / / G$ denotes the differential of $u$. Observe that each $\mathcal{X}_{i}$ is a closed $G$-subvariety of $\mathfrak{g}$. Recall that $y \in \mathfrak{g}$ is called regular if its centralizer in $\mathfrak{g}$ is of dimension $\ell$. Then [10, Theorem 10.1], $\operatorname{rk} d_{y} u=\ell$ if and only if $y$ is regular.

We would like to thank D. Panyushev for the proof of the following proposition, which is considerably easier than our original proof.

Proposition 2.1. One has: $\operatorname{codim} \mathcal{X}_{i} \geq \ell-i+2$, for $0 \leq i \leq \ell-1$.

Proof. Notice that $u$ induces a surjective morphism $\varpi: X_{i} \rightarrow X_{i} / / G$ and that, for all $x \in X_{i}$, the differential $d_{x} \varpi$ is the restriction of $d_{x} u$ to $T_{x} X_{i}$. Set $r=$ $\max \left\{\operatorname{rk} d_{x} \varpi: x \in X_{i}\right\}$. Then, by [7, Proposition III.10.6] and the definition of $X_{i}$, we obtain that $\operatorname{dim} \mathcal{X}_{i} / / G \leq r \leq i$.

Since $X_{i}$ is stable under the $\mathbb{C}^{*}$-action $y \mapsto \lambda y, \lambda \in \mathbb{C}^{*}$, the point 0 belongs to each irreducible component of $X_{i}$. Hence, $\operatorname{dim} X_{i} \leq \operatorname{dim} X_{i} / / G+\operatorname{dim} \varpi^{-1}(\varpi(0))$ 
(see [11, AI.3.3] or [7, Ex. II.3.22]). But $\varpi^{-1}(\varpi(0))=X_{i} \cap \mathbf{N}$, where $\mathbf{N}$ denotes the nilpotent cone of $\mathfrak{g}$, and, since $i \leq \ell-1, X_{i} \cap \mathbf{N}$ is contained in the subvariety of non-regular nilpotent elements. Therefore $\operatorname{dim} \varpi^{-1}(\varpi(0)) \leq n-\ell-2$ and it follows that $\operatorname{dim} X_{i} \leq i+n-\ell-2$, as required.

Remark. Proposition 2.1 generalizes the well-known fact that $X_{\ell-1}$ has codimension at least three (see, for example, [18, Theorem 4.12]). It is natural to conjecture that Proposition 2.1 can be improved to the statement that codim $X_{i} \geq 3(\ell-i)$ for $0 \leq i \leq \ell-1$. D. Panyushev informs us that he has been able to prove this by a case by case analysis.

Fix a $G$-invariant, non-degenerate, symmetric bilinear form $\kappa$ on $\mathfrak{g}$ and let $\tilde{\kappa}: \mathfrak{g}^{*} \rightarrow \mathfrak{g}$ be the induced isomorphism. Thus, $\tilde{\kappa}$ induces an isomorphism between differential one-forms on $\mathfrak{g}$ and vector fields on $\mathfrak{g}$. If $f \in \mathcal{O}^{G}$, then we define a $G$ invariant vector field $\operatorname{grad}(f) \in \mathcal{O} \otimes_{\mathbb{C}} \mathfrak{g}$ to be the image of $d f$ under $\widetilde{\kappa}$. Equivalently, if we fix an orthonormal basis $\left\{e_{i}\right\}$ of $\mathfrak{g}$ and write $x_{i}=e_{i}^{*} \in \mathfrak{g}^{*}$, then

$$
\operatorname{grad}(f)=\sum_{j=1}^{n} \frac{\partial f}{\partial x_{j}} \otimes e_{j}=\sum_{j=1}^{n} \frac{\partial f}{\partial x_{j}} \frac{\partial}{\partial x_{j}} .
$$

By Chevalley's Theorem, $\mathcal{O}^{G}$ is a polynomial ring, say $\mathcal{O}^{G}=\mathbb{C}\left[u_{1}, \ldots, u_{\ell}\right]$ for homogeneous, algebraically independent polynomials $\left\{u_{i}\right\}_{i}$. Set $\nabla_{i}=\operatorname{grad}\left(u_{i}\right)$, for $1 \leq i \leq \ell$. If $\tau: \mathfrak{g} \rightarrow \operatorname{Der} \mathcal{O}$ is the differential of the adjoint action of $G$ on $\mathfrak{g}$, then write $E=\mathcal{O} \tau(\mathfrak{g})$. We will also write $\tau$ for the induced map:

$$
\tau: \mathcal{O} \otimes_{\mathbb{C}} \mathfrak{g} \longrightarrow E \subseteq \operatorname{Der} \mathcal{O} .
$$

Notice that if $\theta \in \mathcal{O} \otimes_{\mathbb{C}} \mathfrak{g}$, the vector field $\tau(\theta)$ is given by $\tau(\theta)_{y}=\left[y, \theta_{y}\right]$ for all $y \in \mathfrak{g}$. It follows easily that if $\theta$ is $G$-invariant, then $\tau(\theta)=0$. In particular, one has $\tau\left(\nabla_{i}\right)=0$ for all $i$. In fact rather more is true:

Lemma 2.2. There is a short exact sequence

$$
0 \longrightarrow \bigoplus_{i=1}^{\ell} \mathcal{O} \nabla_{i} \longrightarrow \mathcal{O} \otimes_{\mathbb{C}} \mathfrak{g} \stackrel{\tau}{\longrightarrow} E \longrightarrow 0
$$

Proof. This is [16, Theorem 2.5.4]. Using the identification of $\mathfrak{g}$ with $\mathfrak{g}^{*}$ under $\tilde{\kappa}$, it also follows from [14, Theorem 1.9].

Corollary 2.3. If $\operatorname{Sym}_{\mathcal{O}}(E)$ denotes the symmetric algebra of the $\mathcal{O}$-module $E$, then, $\operatorname{Sym}_{\mathcal{O}}(E) \cong \operatorname{Sym}_{\mathcal{O}}\left(\mathcal{O} \otimes_{\mathbb{C}} \mathfrak{g}\right) /\left(\nabla_{1}, \ldots, \nabla_{\ell}\right)$.

Proof. This follows from the universal property of symmetric algebras.

Set $\operatorname{Sym}(E)=\operatorname{Sym}_{\mathcal{O}}(E)$. The main aim of this section is to understand the structure of $\operatorname{Sym}(E)$, for which we use the results from [1] and [8]. 
Let $I_{t}(\mathbf{u})$ be the ideal generated by the $t \times t$ minors of the matrix $\mathbf{u}=\left[\frac{\partial u_{i}}{\partial x_{j}}\right]$ and consider the following condition for $s \geq 0$ :

$$
\text { ht } I_{t}(\mathbf{u}) \geq \ell-t+1+s, \quad \text { for } 1 \leq t \leq \ell .
$$

Observe that, if we regard the short exact sequence (2.2) as a sequence

$$
0 \longrightarrow \mathcal{O}^{\ell} \stackrel{\beta}{\longrightarrow} \mathcal{O}^{n} \longrightarrow E \longrightarrow 0,
$$

then (2.1) implies that $I_{t}(\mathbf{u})$ is the ideal generated by the $t \times t$ minors of the map $\beta$. Thus, the ideals $I_{n-t}(\mathbf{u})$ are nothing more than the Fitting ideals of $E$ (see, for example, $[17,1.1])$. In particular, they are independent of the presentation of $E$ and our condition $\left(\mathcal{F}_{s}\right)$ coincides with that of $[8]$.

Proposition 2.4. (i) The condition $\left(\mathcal{F}_{2}\right)$ is satisfied by E.

(ii) $\operatorname{Sym}(E)$ is a factorial domain of Krull dimension $2 n-\ell$. In particular, $\operatorname{Sym}(E)$ is a complete intersection and is Gorenstein.

(iii) If $P$ is a prime ideal of $\mathcal{O}$ with ht $P \geq 2$, then ht $P \operatorname{Sym}(E) \geq 2$.

Proof. Write $\widetilde{X}_{i-1}$ for the zero set of $I_{i}(\mathbf{u})$; thus

$$
\widetilde{x}_{i-1}=\left\{x \in \mathfrak{g}: \operatorname{rk}\left(\nabla_{1}(x), \ldots, \nabla_{\ell}(x)\right) \leq i-1\right\} .
$$

Since the $\nabla_{j}$ are the images of the $d u_{j}$ under the isomorphism $\tilde{\kappa}$, clearly $\widetilde{X}_{i-1}=$ $\left\{x \in \mathfrak{g}: \operatorname{rk}\left(d_{x} u_{1}, \ldots, d_{x} u_{\ell}\right) \leq i-1\right\}$. Since $u_{1}, \ldots, u_{\ell}$ define the quotient map $u: \mathfrak{g} \rightarrow \mathfrak{g} / / G$, this implies that $\widetilde{X}_{i}=X_{i}$. Hence, part (i) is a reformulation of Proposition 2.1.

By Lemma 2.2, $E$ has projective dimension at most 1. Thus, part (ii) follows from part (i), combined with [1, Propositions 3 and 6]. By [8, Remarks, pp. 664-5], the condition of part (iii) is equivalent to the condition $\left(\mathcal{F}_{2}\right)$.

We end this section by giving the geometric significance of Proposition 2.4. This should be compared with $[9, \S 2]$ which proves weaker results for much more general $G$-varieties.

The map $\tau$ induces a homomorphism of algebras

$$
\tilde{\tau}: \mathcal{O}\left(\mathfrak{g} \times \mathfrak{g}^{*}\right)=\operatorname{Sym}_{\mathcal{O}}(\mathcal{O} \otimes \mathfrak{g}) \longrightarrow \mathcal{O}\left(T^{*} \mathfrak{g}\right)=\operatorname{Sym}_{\mathcal{O}}(\operatorname{Der} \mathcal{O}) .
$$

Clearly, the image of $\tilde{\tau}$ is the subring $\mathcal{O}[\tau(\mathfrak{g})]$ of $\operatorname{Sym}_{\mathcal{O}}(\operatorname{Der} \mathcal{O})$ generated by $\mathcal{O}$ and $\tau(\mathfrak{g})$. After identification of $\mathfrak{g}^{*}$ with $\mathfrak{g}$ through $\tilde{\kappa}$, the associated morphism to $\tilde{\tau}$ is:

$$
\nu: T^{*} \mathfrak{g} \cong \mathfrak{g} \times \mathfrak{g} \longrightarrow \mathfrak{g} \times \mathfrak{g}, \quad \nu(x, y)=(x,[y, x])
$$

Let $\tilde{T} \mathfrak{g}$ denote the closure of the image of $\nu$; thus, $\tilde{T} \mathfrak{g}$ is an irreducible affine subvariety of $\mathfrak{g} \times \mathfrak{g}$ with coordinate ring $\mathcal{O}(\tilde{T} \mathfrak{g}) \cong \mathcal{O}[\tau(\mathfrak{g})]$. 
Corollary 2.5. (i) $\operatorname{Sym}(E)=\mathcal{O}(\tilde{T} \mathfrak{g})$.

(ii) The variety $\tilde{T} \mathfrak{g}$ is a factorial complete intersection in $\mathfrak{g} \times \mathfrak{g}$.

Proof. By universality, $\tilde{\tau}$ induces a surjective morphism $\pi: \operatorname{Sym}(E) \rightarrow \mathcal{O}[\tau(\mathfrak{g})]$. If we prove that $\operatorname{dim} \tilde{T} \mathfrak{g} \geq 2 n-\ell$, then the corollary will follow from Proposition 2.4(ii).

Let $\rho: \tilde{T} \mathfrak{g} \rightarrow \mathfrak{g}$ denote the projection onto the first factor. By [11, AI.3.3] there exists a dense open subset $U \subseteq \tilde{T} \mathfrak{g}$ such that $\operatorname{dim} \tilde{T} \mathfrak{g}=\operatorname{dim} \mathfrak{g}+\operatorname{dim} \rho^{-1}(\rho(u))$ for all $u \in U$. Since $\tilde{T} \mathfrak{g}$ is irreducible, we can pick $u=(x, y) \in \rho^{-1}\left(\mathfrak{g}^{\prime}\right) \cap U$, where $\mathfrak{g}^{\prime}$ denotes the set of generic elements in $\mathfrak{g}$. Now,

$$
\rho^{-1}(\rho(u)) \supseteq \rho^{-1}(\rho(u)) \cap \operatorname{Im} \nu=\{(x,[\mathfrak{g}, x])\} .
$$

Since $x$ is generic, $\operatorname{dim} \rho^{-1}(\rho(u)) \geq \operatorname{dim}[\mathfrak{g}, x]=n-\ell$ and the result follows.

\section{The commutant of $\mathcal{O}(\mathfrak{g})^{G}$}

As usual, we identify $\mathcal{D}(\mathfrak{g})$, as a vector space, with $\mathcal{O} \otimes_{\mathbb{C}} S(\mathfrak{g})$, where $\mathcal{O}=\mathcal{O}(\mathfrak{g})$ and the symmetric algebra $S(\mathfrak{g})$ is identified with the constant coefficient differential operators on $\mathfrak{g}$. We will always filter $\mathcal{D}(\mathfrak{g})$ by degree of differential operators and so, as algebras, $\operatorname{gr} \mathcal{D}(\mathfrak{g})=\operatorname{Sym}_{\mathcal{O}}(\operatorname{Der} \mathcal{O}) \cong \mathcal{O} \otimes_{\mathbb{C}} S(\mathfrak{g})$. Write $A$ for the subring of $\mathcal{D}(\mathfrak{g})$ generated by $\mathcal{O}$ and $\tau(\mathfrak{g})$ and let $\mathcal{C}$ denote the commutant of $\mathcal{O}^{G}$, as in the introduction. Obviously, $A$ is contained in $\mathrm{C}$.

Lemma 3.1. Let $x \in \mathfrak{g}$ be a regular point and set $R=\mathcal{O}_{\mathfrak{g}, x}$ for the local ring of $\mathfrak{g}$ at $x$. Then, there exists a basis of derivations $\left\{\partial_{i}: 1 \leq i \leq n\right\}$ of Der $R$ such that $\partial_{i}\left(u_{j}\right)=\delta_{i j}$ for all $1 \leq i, j \leq \ell$ and $R \tau(\mathfrak{g})=\bigoplus_{i=\ell+1}^{n} R \partial_{i}$.

Proof. Let $\mathbf{m}$ denote the maximal ideal of $R$. By [10, Theorem 0.1], the $\left\{d_{x} u_{i}\right.$ : $1 \leq i \leq \ell\}$ are linearly independent. The $\left\{d_{x} u_{i}\right\}$ may also be regarded as elements of $\mathbf{m} / \mathbf{m}^{2}$, under the usual identification of $T_{x}^{*} \mathfrak{g}$ with $\mathbf{m} / \mathbf{m}^{2}$. Thus, for some scalars $\lambda_{i}$, the set $\left\{u_{1}-\lambda_{1}, \ldots, u_{\ell}-\lambda_{\ell}\right\}$ is part of a system of parameters, say $\left\{z_{1}=u_{1}-\lambda_{1}, \ldots, z_{\ell}=u_{\ell}-\lambda_{\ell}, z_{\ell+1}, \ldots, z_{n}\right\}$ for $\mathbf{m}$. Let $\partial_{i} \in$ Der $R$ be defined by $\partial_{i}\left(z_{j}\right)=\delta_{i j}$.

If $D \in \operatorname{Der} R$, then $\widetilde{D}=D-\sum_{i=1}^{\ell} D\left(u_{i}\right) \partial_{i}$ satisfies $\widetilde{D}\left(u_{j}\right)=0$, for $1 \leq j \leq \ell$. Thus, $\widetilde{D}\left(\mathcal{O}^{G}\right)=0$ and so, by [5, Theorem 2.1] (or directly), $\widetilde{D} \in R \tau(\mathfrak{g})$. Hence, Der $R=R \tau(\mathfrak{g}) \oplus\left(\oplus_{i=1}^{\ell} R \partial_{i}\right)$. Since

$$
R \tau(\mathfrak{g}) \subseteq\left\{D \in \operatorname{Der} R: D\left(u_{j}\right)=0 \text { for } 1 \leq j \leq \ell\right\}=\bigoplus_{i=\ell+1}^{n} R \partial_{i},
$$

the result follows.

Theorem 3.2. Let $A$ and $\mathcal{C}$ be given the filtrations induced from that on $\mathcal{D}(\mathfrak{g})$. Then $\operatorname{gr} A=\operatorname{gr} \mathcal{C} \cong \operatorname{Sym}(E)$. 
Proof. Since gr $\mathcal{C} \subset \operatorname{gr} \mathcal{D}(\mathfrak{g}) \cong \mathcal{O}\left(T^{*} \mathfrak{g}\right)$, certainly gr $A \subseteq \operatorname{gr} \mathcal{C}$ are domains. Also, as $\tau(\mathfrak{g})$ consists of derivations, we may regard $\tau(\mathfrak{g}) \subseteq \operatorname{Der} \mathcal{O} \subseteq \operatorname{gr} \mathcal{D}(\mathfrak{g})$. Hence the ring $\mathcal{O}[\tau(\mathfrak{g})]$ is contained in gr $A$ and, by Corollary 2.5(i), the natural map $\pi: \operatorname{Sym}(E) \rightarrow \mathcal{O}[\tau(\mathfrak{g})]$ is an isomorphism.

Let $x \in \mathfrak{g}$ be a regular point and let $\mathcal{S}=\{f \in \mathcal{O}: f(x) \neq 0\}$. Given a ring $C$ containing $\mathcal{O}$, we write $C_{x}$ for the localization $C_{\mathcal{S}}$ (given that it exists). Then, we claim that

$$
(\operatorname{gr} A)_{x}=(\operatorname{gr} \mathcal{C})_{x} \cong \operatorname{Sym}(E)_{x}
$$

where the isomorphism is induced by $\pi^{-1}$.

By mimicking the proof of Richardson's Lemma [11, II.3.4], one can show that this suffices to prove the theorem. In more detail, assume that (3.1) is true. Since gr $\mathcal{C}$ and $\operatorname{Sym}(E)$ are domains, (3.1) certainly implies that

$$
\operatorname{Sym}(E) \stackrel{\pi}{\hookrightarrow} \operatorname{gr} A \subseteq \operatorname{gr} \mathcal{C}
$$

and that $\operatorname{gr} \mathcal{C}$ and $\operatorname{Sym}(E)$ have the same field of fractions. Moreover, $\{x \in \mathfrak{g}$ : $\left.(\operatorname{gr} \mathcal{C})_{x} \neq \operatorname{Sym}(E)_{x}\right\}$ is contained in the set of non-regular elements of $\mathfrak{g}$. By [10, Theorem 0.1], this is precisely the subspace $X_{\ell-1}$ and, by Proposition 2.1 or [18, Theorem 4.12], codim $X_{\ell-1} \geq 3$. Thus, for any $b \in \operatorname{gr} \mathcal{C}$, there exists an ideal $I$ of $\mathcal{O}$ of height at least 3 such that $b I \subseteq \operatorname{Sym}(E)$. By Proposition 2.4(iii), $\mathrm{ht}_{\operatorname{Sym}(E)} I \operatorname{Sym}(E) \geq 2$. Hence, $b \in \operatorname{Sym}(E)_{\mathbf{p}}$ for every height one prime $\mathbf{p}$ of $\operatorname{Sym}(E)$. Since $\operatorname{Sym}(E)$ is Cohen-Macaulay, it satisfies the $\left(\mathrm{S}_{2}\right)$-condition [12, p. 125], and therefore $b \in \operatorname{Sym}(E)$.

Thus, it remains to prove (3.1). Let $R=\mathcal{O}_{x}=\mathcal{O}_{\mathfrak{g}, x}$ and keep the notation of Lemma 3.1. It is immediate from that lemma that $D \in \mathcal{D}(\mathfrak{g})_{x}$ satisfies $\left[D, u_{j}\right]=0$ if and only if $D \in R\left\langle\partial_{j}: j \neq i\right\rangle$. Consequently, $\mathcal{C}_{x}=A_{x}=R\left\langle\partial_{\ell+1}, \ldots, \partial_{n}\right\rangle$.

Let $\bar{\partial}_{k}$ denote the image of $\partial_{k}$ in gr $\mathcal{D}(\mathfrak{g})$. Obviously, Lemma 3.1 also implies that $R \tau(\mathfrak{g})=\bigoplus_{k=\ell+1}^{n} R \bar{\partial}_{k}$, where $R \tau(\mathfrak{g})$ is now regarded as a subspace of Der $R \subset$ $\operatorname{gr} \mathcal{D}(\mathfrak{g})_{x} \cong R \otimes_{\mathbb{C}} S(\mathfrak{g})$. Thus,

$$
\operatorname{gr} \mathcal{C}_{x}=\operatorname{gr} A_{x}=\operatorname{gr} R\left\langle\partial_{\ell+1}, \ldots, \partial_{n}\right\rangle=R\left[\bar{\partial}_{\ell+1}, \ldots, \bar{\partial}_{n}\right]=R[\tau(\mathfrak{g})] .
$$

Since $\pi: \operatorname{Sym}(E)_{x} \rightarrow R[\tau(\mathfrak{g})]$ is an isomorphism, this completes the proof of (3.1) and hence of the theorem.

The Gelfand-Kirillov dimension of a module $M$ will be denoted GKdim $M$. If a Noetherian ring $A$ has finite injective dimension, then $A$ is called AuslanderGorenstein if $A$ satisfies the following condition: For any integers $0 \leq i<j$ and finitely generated (right) $A$-module $M$, one has $\operatorname{Ext}_{A}^{i}(N, A)=0$ for all (left) $A$-submodules $N$ of $\operatorname{Ext}_{A}^{j}(M, A)$. Set j $(M)=\min \left\{j: \operatorname{Ext}_{A}^{j}(M, A) \neq 0\right\}$. The algebra $A$ is $C M$ if $\mathrm{j}(M)+\operatorname{GK} \operatorname{dim} M=\operatorname{GKdim} A$ holds for all finitely generated, non-zero $A$-modules $M$.

Corollary 3.3. (i) The commutant $\mathcal{C}$ of $\mathcal{O}^{G}$ in $\mathcal{D}(\mathfrak{g})$ is the ring generated by $\mathcal{O}$ and $\tau(\mathfrak{g})$. Moreover, $\mathcal{C}$ is an Auslander-Gorenstein, CM, Noetherian domain and a maximal order. 
(ii) As a (left or right) $\mathcal{O}$-module, $\mathcal{C} \cap \mathcal{D}(\mathfrak{g})_{m}$ is generated by the elements

$$
\left\{\tau\left(\xi_{1}\right) \tau\left(\xi_{2}\right) \cdots \tau\left(\xi_{k}\right): \xi_{i} \in \mathfrak{g} \text { and } k \leq m\right\} .
$$

(iii) The centre of $\mathcal{C}$ is $\mathcal{O}(\mathfrak{g})^{G}$.

Proof. (i) By Theorem 3.2, $\mathrm{C}$ is generated by $\mathcal{O}$ and $\tau(\mathfrak{g})$. By that theorem and Proposition 2.4, gr $\mathrm{C}$ satisfies the other conditions given in part (i). Let $M=$ $\bigcup_{n \in \mathbb{N}} M_{n}$ be a filtered right $\mathcal{C}$-module such that gr $M$ is a finitely generated gr $\mathcal{C}$ module. By [3, Theorem 3.9], $\mathcal{C}$ is Auslander-Gorenstein and $\mathrm{j}_{\mathcal{C}}(M)=\mathrm{j}_{\operatorname{gr} \mathcal{C}}(\operatorname{gr} M)$. However, [13, Corollary 1.4] implies that GKdimgr $M=\operatorname{GKdim} M$ and hence that $\mathcal{C}$ is $\mathrm{CM}$. Finally, [15] implies that $\mathcal{C}$ is a maximal order.

(ii) This follows from the fact that, in $\operatorname{gr} \mathcal{C}=\operatorname{Sym}(E)$, a homogeneous element $\bar{c}$ of degree $m$ can be written $\bar{c}=\sum f_{i_{1}, \ldots, i_{m}} \xi_{i_{1}} \cdots \xi_{i_{m}}$, for some $f_{i_{1}, \ldots, i_{m}} \in \mathcal{O}$ and $\xi_{i_{j}} \in \tau(\mathfrak{g})$.

(iii) Let $Z$ denote the centre of $\mathcal{C}$. Clearly both $\tau(\mathfrak{g})$ and $\mathcal{O}$ commute with $\mathcal{O}^{G}$ and so $\mathcal{O}^{G} \subseteq Z$. Conversely, $Z$ is contained in the commutant, in $\mathcal{D}(\mathfrak{g})$, of $\mathcal{O}$. Hence, $Z \subseteq \mathcal{O}$. Since $\mathcal{O}^{G}$ is the commutant, in $\mathcal{O}$, of $\tau(\mathfrak{g})$, the result follows.

Corollary 3.4. Both $\mathcal{C}$ and $\operatorname{Sym}(E)$ are free (left or right) modules over $\mathcal{O}(\mathfrak{g})^{G}$.

Proof. Set $\mathcal{O}=\mathcal{O}(\mathfrak{g})$ and $S=\operatorname{Sym}(E)=\bigoplus_{m=0}^{\infty} \operatorname{Sym}_{m}(E)$. We first prove the result for $\mathcal{C}$, assuming that $\operatorname{Sym}_{m}(E)$ is a free $\mathcal{O}^{G}$-module for all $m \in \mathbb{N}$. Note that the isomorphism gr $\mathcal{C} \cong S$ of Theorem 3.2 is a graded isomorphism of $\mathcal{O}$ algebras, for the natural graded structure of the two objects. In other words $\mathcal{C}_{m} / \mathcal{C}_{m-1} \cong \operatorname{Sym}_{m}(E)$, for all $m$, where $\mathcal{C}_{m}=\mathcal{C} \cap \mathcal{D}(\mathfrak{g})_{m}$. Hence, each $\mathcal{C}_{m} / \mathcal{C}_{m-1}$ is a free $\mathcal{O}^{G}$-module; it follows routinely that $\mathcal{C}$ is also free over $\mathcal{O}^{G}$.

We now prove the result for $\operatorname{Sym}_{m}(E)$. Note, first, that $S$ is a quotient of the polynomial ring

$$
T=\operatorname{Sym}_{\mathcal{O}}(\mathcal{O} \otimes \mathfrak{g}) \cong \mathcal{O}\left[y_{1}, \ldots, y_{n}\right] \cong \mathbb{C}\left[x_{1}, \ldots, x_{n}, y_{1} \ldots, y_{n}\right],
$$

which we now grade by giving each generator $x_{i}$ and $y_{j}$ degree one. Since the $u_{i}$ are homogeneous in $\mathcal{O}$, the $\nabla_{i}=\operatorname{grad}\left(u_{i}\right)$ are homogeneous in $T$ and so, by Corollary 2.3, $\operatorname{Sym}_{m}(E)$ is a graded $\mathcal{O}^{G}$-module.

Set $P=\sum_{i=1}^{\ell} u_{i} S$. By [6, Proposition 2.16] and its proof (which depends upon a case by case analysis), $S / P$ is a domain of dimension $2 n-2 \ell=\operatorname{dim} S-\ell$. Hence, the $u_{j}$ form a regular sequence in $S$, and therefore in each module $\operatorname{Sym}_{m}(E)$. Thus, by $\left[4, \S 8\right.$, Proposition 8 and $\S 9$, Corollaire 2], $\operatorname{Sym}_{m}(E)$ is a graded free $\mathcal{O}^{G}$ module.

Corollary 3.3 and Corollary 3.4 should be compared with [9] which (as a very special case) shows that the commutant of $\mathcal{D}(\mathfrak{g})^{G}$ is simply $\mathbb{C}\langle\tau(\mathfrak{g})\rangle(\cong U(\mathfrak{g})$ when $\mathfrak{g}$ is semisimple). Moreover, both rings are free modules over the centre of $\mathcal{D}(\mathfrak{g})^{G}$ (which is also the centre of $\mathbb{C}\langle\tau(\mathfrak{g})\rangle$ ). 


\section{References}

[1] L. L. Avramov, Complete intersections and symmetric algebras, J. Algebra 73 (1981), 248263.

[2] D. Barlet, Unpublished manuscript, Université de Nancy, 1978.

[3] J.-E. Björk, The Auslander condition on Noetherian rings. In: Séminaire d'Algèbre P. Dubreil et M.-P. Malliavin, Lecture Notes in Math. 1404, Springer Verlag, Berlin/New York 1989, 137-173.

[4] N. Bourbaki, Algèbre, Chapitre 10, Masson, Paris 1980.

[5] J. Dixmier, Champs de vecteurs adjoints sur les groupes et algèbres de Lie semi-simples, J. Reine Angew. Math. 309 (1979), 183-190.

[6] F. Geoffriau, Sur le centre de l'algèbre enveloppante d'une algèbre de Takiff, Ann. Math. Blaise Pascal 1 (1994), 15-31.

[7] R. Hartshorne, Algebraic Geometry, Graduate Texts in Math., Vol. 52, Springer-Verlag, Berlin/New York 1977.

[8] J. Herzog, A. Simis and W. V. Vasconcelos, On the arithmetic and homology of algebras of linear type, Trans. Amer. Math. Soc. 321 (1990), 183-196.

[9] F. Knop, A Harish-Chandra homomorphism for reductive group actions, Ann. Math., 140 (1994), 253-288.

[10] B. Kostant, Lie group representations on polynomial rings, Amer. J. Math. 85 (1963), 327-404.

[11] H. Kraft, Geometrische Methoden in der Invariantentheorie, Aspekte der Mathematik, Vieweg, Braunschweig 1994.

[12] H. Matsumura, Commutative Algebra (Second Edition), Benjamin Publ. Co., Reading 1980.

[13] J. C. McConnell and J. T. Stafford, Gelfand-Kirillov dimension and associated graded modules, J. Algebra 125 (1989), 197-214.

[14] D. Panyushev, The Jacobian modules of a representation of a Lie algebra and geometry of commuting varieties, Compositio Math. 94 (1994), 181-199.

[15] M. Van den Bergh and F. Van Oystaeyen, Lifting maximal orders, Comm. in Algebra 17 (1989), 341-349.

[16] W. V. Vasconcelos, Symmetric algebras. In: Commutative Algebra, Salvador, 1988, Lecture Notes in Math. 1430, Springer-Verlag, Berlin/New York 1990, 115-160.

[17] W. V. Vasconcelos, Arithmetic of Blowup Algebras, Cambridge University Press, 1994.

[18] F. D. Veldkamp, The center of the universal enveloping algebra of a Lie algebra in characteristic p, Ann. Sci. Ecole Norm. Sup. 5 (1972), 217-240.

T. Levasseur

Département de Mathématiques

Université de Poitiers

F-86022 Poitiers, France

e-mail: levasseu@mathlabo.univ-poitiers.fr
Department of Mathematics

University of Michigan

Ann Arbor, MI 48109, USA

e-mail: jts@math.lsa.umich.edu

(Received: July 3, 1996) 